\title{
MiRNA-29c-3p Promotes Intestinal Inflammation via Targeting Leukemia Inhibitory Factor in Ulcerative Colitis
}

Jian Guo ${ }^{1,2}$

Ruiya Zhang ${ }^{3}$

Yiqing Zhao ${ }^{3}$

Junping Wang ${ }^{3}$

'Key Laboratory of Chemical Biology and Molecular Engineering of Ministry of Education, Shanxi University, Taiyuan, 030006, People's Republic of China; ${ }^{2}$ Department of General Surgery, Shanxi Provincial People's Hospital, The Affiliated People's Hospital of Shanxi Medical University, Taiyuan, 030012 , People's Republic of China; ${ }^{3}$ Department of Gastroenterology, Shanxi Provincial People's Hospital, The Affiliated People's Hospital of Shanxi Medical University, Taiyuan, Shanxi, 0300I2, People's Republic of China
Correspondence: Junping Wang Department of Gastroenterology, Shanxi Provincial People's Hospital, The Affiliated People's Hospital of Shanxi Medical University, 29 Shuangtasi Road, Taiyuan, Shanxi, 0300 12, People's Republic of China

Tel + 35I-4960I30

Email wermik527@2Icn.com
Background: Dysregulation of micro-RNAs (miRNAs) is profoundly linked to inflammatory bowel diseases (IBD), but little is known about the specific biological functions of miRNAs in IBD. This study sought to elucidate the effect and the underlying target of miR$29 \mathrm{c}-3 \mathrm{p}$ in ulcerative colitis (UC).

Methods: The levels of miR-29c-3p and leukemia inhibitory factor (LIF) were measured in inflamed lesions of UC patients and dextran sulfate sodium (DSS)-induced colitis mice by quantitative real-time polymerase chain reaction (qRT-PCR) and Western blotting. MiR-29c$3 p$ was predicted to target LIF by bioinformatics software, which was verified via luciferase reporter assay and transfection of miR-29c-3p mimics or inhibitor. The role of miR-29c-3p/ LIF axis in intestinal inflammation was explored in experimental colitis mice and Caco-2 cells.

Results: MiR-29c-3p was markedly downregulated while LIF was upregulated in colon tissues of UC patients and DSS-challenged colitis mice as well as in primary intestinal epithelial cells (IECs) and LPS-treated Caco-2 cells. MiR-29c-3p inhibited LIF expression at the transcriptional level via binding to LIF 3 '-untranslated region (UTR) in Caco-2 cells. Targeting miR-29c-3p/LIF axis regulated inflammatory cytokines production, cell proliferation and apoptosis. Overexpression of miR-29c-3p aggravated mice experimental colitis via suppressing LIF.

Conclusion: Our findings demonstrate that the upregulation of miR-29c-3p promotes gut inflammation and the expression of pro-inflammatory mediators via suppressing LIF, thereby modulating the pathogenesis of UC.

Keywords: ulcerative colitis, microRNA-29c-3p, leukemia inhibitory factor, inflammation, apoptosis

\section{Introduction}

Ulcerative colitis (UC), one of the two primary types of inflammatory bowel diseases (IBD), refers to chronic immune-mediated disorders featured by chronic and recurrent gut inflammation. Although the exact etiology and pathology remain elusive, it is generally believed that UC is a multifactorial disorder that includes environmental trigger such as diet, lifestyle and a maladaptive immune response to gut-resident commensal bacteria in a genetically susceptible host. ${ }^{1}$ Notably, cytokines mediating the balance of immune system play a pivotal role in maintaining the homeostasis of the gastrointestinal tract. ${ }^{2}$ Overexpression of pro-inflammatory mediators, such as interleukin (IL)-1 $\beta$, IL-6, tumor necrosis factor (TNF)- $\alpha$, has been considered to be 
closely associated with the onset of gut inflammation as well as the persistence tissue damage. ${ }^{3}$ Despite that both Crohn's disease (CD) and UC are not life-threatening, none of them are curable, and the present therapeutic options still aim at achieving remission and improvement of the symptoms. ${ }^{4}$ Consequently, exploring novel biomarkers and treatment targets for IBD remains a pressing priority.

As a class of newly identified small non-coding RNAs $(\sim 22$ nucleotides), micro-RNAs (miRNAs) interact with target messenger RNAs (mRNAs) at the post-transcriptional level, participating in various pathophysiology processes, including cell proliferation, apoptosis, regulation of immune response and tissue homeostasis. ${ }^{5,6}$ Given their extensive biological functions, miRNAs have been implicated in multiple human diseases, including cancers and inflammatory diseases, such as IBD. ${ }^{7-11}$ Since the genetic factors are crucial in the pathogenesis of IBD, the relationship between miRNAs and IBD has raised broad attention recently. For example, Tian et al found that increased miR-31 in inflamed colon tissues of IBD attenuates mice colitis by reducing inflammatory signaling and promoting regeneration of colon epithelium. ${ }^{12}$ It was also reported that miR-301a was upregulated in inflamed mucosa of IBD and promoted inflammatory response in experimental mice colitis. $^{13}$ Therefore, promising insights into miRNAs in IBD may give rise to potential diagnostic biomarkers and therapies.

Leukemia inhibitory factor (LIF), a member of IL-6 family, is a pleiotropic cytokine characterized by its paradoxically opposite effects in different cells. ${ }^{14}$ LIF acts on multiple cell types by binding to its receptor, which consists of two signaling chains: LIF receptor $\beta$ and gp130. ${ }^{14}$ Although it was reported that the expression of LIF in the colonic mucosa of UC patients was increased ${ }^{15}$ and STAT4 activation by LIF exerted a therapeutic effect on gut inflammation in mice colitis model, ${ }^{16}$ little is known about the regulation of LIF in colonic inflammation.

Previous studies revealed that miR-29c-3p was involved in the pathogenesis of colorectal cancer, ${ }^{17}$ but the role of miR-29c-3p in IBD remains largely unknown. In this study, we sought to analyze the expression of miR$29 \mathrm{c}-3 \mathrm{p}$ in the lesions of UC patients and investigated whether miR-29c-3p regulated intestinal inflammation via targeting LIF in vivo and in vitro.

\section{Materials and Methods}

\section{Patients}

Tissue samples were collected from 20 UC patients and 20 normal control individuals undergoing screening colonoscopies or surgery at Shanxi Provincial People's Hospital. All the samples were stored in liquid nitrogen before experiments. Patients with UC were diagnosed by experienced physicians according to the findings of colonoscopies and biopsies. All human experiments were approved by the Ethics Committee of Shanxi Provincial People's Hospital (Number: 202065) and conducted in conformity to the Declaration of Helsinki. Written informed consent was also obtained from each participant.

\section{Isolation of Primary Intestinal Epithelial Cells (IECs)}

Primary IECs from human/mouse colon were sorted under cryogenic conditions using chelating agents, as previously described. ${ }^{18}$ In brief, colon tissues were separated, washed and cut into small pieces in cold phosphate-buffered saline (PBS), followed by gently shaking in PBS containing 2 $\mathrm{mmol} / 1 \mathrm{DTT}$ and $1 \mathrm{mmol} / 1 \mathrm{EDTA}$ at $37^{\circ} \mathrm{C}$ to isolate IECs. For further purification, the cells were harvested and purified by density gradient centrifugation with 20 and $40 \%$ percoll-RPMI solution.

\section{Cell Culture}

The human colon epithelial cell-line Caco-2 was purchased from American Type Culture Collection (Manassas, VA, USA), and cultured in Dulbecco's modified Eagle's medium (Gibco, MD, USA) supplemented with 10\% fetal bovine serum (Gibco, MD, USA), $100 \mathrm{U} / \mathrm{mL}$ penicillin, and $100 \mu \mathrm{g} / \mathrm{mL}$ streptomycin (Gibco, MD, USA). The cells were incubated at $37^{\circ} \mathrm{C}$ in a $5 \% \mathrm{CO}_{2}$ atmosphere and treated with $1 \mathrm{ug} / \mathrm{mL}$ lipopolysaccharide (LPS, Sigma-Aldrich) for 24 hours.

\section{Cell Transfection}

Synthetic miR-29c-3p mimic, miR-29c-3p inhibitor and scrambled negative control (NC) RNA (miR-NC and inhibitor NC) were obtained from GenePharma (Shanghai, China). A small interfering RNA (siRNA) targeting human LIF gene was purchased from Ribobio (Guangzhou, China). Caco-2 cells were seeded uniformly in 6-well plates and transfected with miR-29c-3p mimic, miR-29c-3p inhibitor, si-LIF or their corresponding negative control RNA the following day using Lipofectamine 2000 (Invitrogen, USA). Then, the pre-transfected cells were stimulated with LPS or left untreated. The cells and the supernatant were harvested for following experiments 24 hours after LPS challenged. 


\section{Target Prediction and Luciferase Reporter Assay}

Several databases were used to predict the potential regulatory miRNA of LIF and the putative target site, including TargetScan (context $^{++}$score percentile $\geq 97$; http://www.tar getscan.org/vert 71/), miRDB (Target Score $\geq 95$; http:// www.mirdb.org/), Very High; http://ophid.utoronto.ca/ mirDIP/), starBase (pancancerNum $\geq 10$; http://starbase. sysu.edu.cn/) and miRmap (miRmap score $>85$; https://mir map.ezlab.org/app/). Then, a luciferase reporter assay was carried out to test the binding of miR-29c-3p to the target gene LIF. DNA fragments of wild-type and mutant LIF 3'untranslated region (UTR) containing the possible miR-29c$3 p$ complementary region were cloned into the luciferase reporter vector. Subsequently, Caco-2 cells were transfected with 3'-UTR or mutant 3'-UTR luciferase reporters only, or along with miR-29c-3p mimic or inhibitor. 48 hours after transfection, cells were collected and assayed for luciferase activity by Luciferase Reporter Assay System (Promega, USA).

\section{Cell Proliferation Assay}

Cell counting kit-8 (CCK-8; Beyotime Institute of Biotechnology) was carried out to determine the proliferation ability of Caco- 2 cells, in accordance with manufacturer's protocol. Untreated or pre-transfected Caco-2 cells were plated on 24-well plates; then, $10 \mathrm{ul} \mathrm{CCK-8} \mathrm{reagent} \mathrm{was}$ added to each well and the optical density was measured every 24 hours for 3 days at a wavelength of $450 \mathrm{~nm}$.

\section{Cell Apoptosis Assay}

Caco-2 cells transfected with miR-29c-3p mimic, miR-29c$3 p$ inhibitor, si-LIF, scrambled control or untreated cells were stained with Annexin V(AV)-FITC/propidium iodide (PI) (Beyotime Institute of Biotechnology). Apoptosis was detected by flow cytometry. The early apoptotic cells (AVFITC positive, PI negative) and late apoptotic cells (AVFITC and PI both positive) were both included.

\section{Experimental Colitis Model and Treatment}

Female C57BL/6 mice (6-8 weeks, 20-22 g) were purchased from Beijing Vital River Laboratory Animal Technologies Co. Ltd (Beijing, China), and were maintained under a specific pathogen-free condition with free access to sterile water and autoclaved food. All animal experiments were conducted with the approval of the Animal Care and
Use Committee of Shanxi Medical University (Number: 202065) and according to the Guide for the Care and Use of Laboratory Animal published by the US National Institutes of Health (NIH publication, Eighth edition, 2011).

Experimental mice colitis was induced by administration with $2.5 \%$ Dextran sulfate sodium (DSS, MP Biomedicals) in the drinking water for consecutive 7 days. Polyetherimide (PEI, $25 \mathrm{kDa}$; Sigma)/miR-29c-3p inhibitor or inhibitor NC complexes were prepared by mixing equal volume of solution of $4 \mathrm{mg} / \mathrm{mL}$ PEI and $2 \mathrm{mg} / \mathrm{mL}$ miR-29c-3p inhibitor or inhibitor NC. The mice were randomly allocated into five groups ( $\mathrm{n}=10$ per group): control group, DSS group, DSS $+\mathrm{PEI}$ group, DSS+PEI/miR-29c-3p inhibitor and DSS+PEI/ inhibitor NC group. Mice were treated intracolonically with equal volume (100ul) of PEI/miR-29c-3p inhibitor, PEI/ inhibitor NC or PEI alone at a dose of $5 \mathrm{mg}$ miR-29c-3p inhibitor $/ \mathrm{kg}$.

Body weight loss and stool property were recorded daily after treatment. The disease activity index (DAI) was used for determination of intestinal inflammation severity according to the method described previously: ${ }^{19}$ body weight loss $(0=$ none; $1=1-5 \% ; 2=6-10 \% ; 3=11-20 \%$; $4=$ $>20 \%$ ), stool consistency $(0=$ normal; $2=$ loose stool; $4=$ diarrhea), and rectal bleeding ( $0=$ negative; $2=$ visible blood in stool; 4=gross bleeding). All the mice were sacrificed on day 8 , and colon tissues were removed and stored for further experiments.

\section{Histopathological Examinations}

Small segments of colon tissues were fixed with $4 \%$ paraformaldehyde for 24 hours at room temperature, and then embedded in paraffin, serially sectioned at 4-um thickness and stained with hematoxylin and eosin (H\&E) for morphological evaluation.

\section{Quantitative Real-Time Polymerase Chain Reaction (qRT-PCR)}

Total RNA was extracted using Trizol (Invitrogen, USA) reagent. qRT-PCR of miR-29c-3p was performed with TaqMan microRNA probes (Thermo Scientific, USA) based on the instructions. The expression of miR-29c-3p was assayed by $\operatorname{TaqMan}^{\circledR}$ small RNA assay kit (Thermo Scientific, USA). The relative miR-29c-3p levels were normalized to $\mathrm{U} 6$ and analyzed with $2^{-\Delta \Delta \mathrm{Ct}}$ method.

For LIF quantification, the extracted RNA was reverse transcribed into cDNA using the PrimeScript ${ }^{\mathrm{TM}}$ RT reagent Kit (Takara, Japan). Quantitative PCR analyses were 
performed on LightCycler ${ }^{\circledR} 480$ System (Roche) using the SYBR Green assay (Takara, Japan). The relative RNA level of LIF was normalized against $\beta$-actin and $2^{-\Delta \Delta \mathrm{Ct}}$ method was used for analysis. Primers used in this study are listed in Table 1 .

\section{Western Blotting}

Total proteins from cells and colon tissues were extracted with RIPA lysis buffer (Beyotime Biotechnology, China) supplemented with protease and phosphatase inhibitor Cocktail (MedChemExpress, China), and were quantified by BCA protein assay kit (Thermo Scientific, USA). Proteins were separated by $10 \%$ sodium dodecyl sulfate polyacrylamide gel (SDS-PAGE) electrophoresis and then transferred onto polyvinylidene difluoride membranes. Membranes were then blocked by a blocking buffer composed of $8 \%$ non-fat milk in Tris buffered saline with $0.1 \%$ Tween-20 for $1 \mathrm{~h}$ at room temperature, followed by incubation with primary anti-LIF antibody (Abcam, UK) and GAPDH antibody (Antgene Biotechnology, China). The protein expression was visualized and quantified by chemiluminescence imaging software (Bio-Rad).

\section{Enzyme Linked Immune-Sorbent Assay (ELISA)}

The cytokines IL-1 $\beta$, IL- 6 , IL- 8, TNF- $\alpha$ in the culture supernatants of pre-transfected Caco- 2 cells and tissue homogenates as prepared above were measured by ELISA kits (R\&D Systems, USA) according to the manufacturer's instructions.

\section{Statistical Analysis}

All data were analyzed and shown as mean \pm S.D based on at least three experiments. The differences between groups were compared by Student's $t$-test or one-way analysis of variance (ANOVA). SPSS 13.0 statistical package software and GraphPad Prism 7.0 were applied for statistical analysis and plotting. A P value $<0.05$ was considered to be statistically significant.

\section{Results}

The Expression of miR-29c-3p and LIF in UC Tissues and Primary IECs

Colonic biopsies were obtained during endoscopic examinations. We first assessed LIF expression in colon tissues using qRT-PCR and found it to be upregulated in UC patients compared with healthy controls (Figure 1A). The enhanced LIF protein expression was then verified by Western blotting (Figure 1B). To further explore cellular level of LIF, primary IECs were isolated and quantified for LIF expression. As expected, the same change trends were observed in IECs (Figure $1 \mathrm{C}$ and D). Next, we adopted five miRNA databases (TargetScan, miRDB, starBase, mirDIP and miRmap) to predict the upstream miRNA of LIF. As a result, miR-29c-3p was the only miRNA with the highest possibility to interact with LIF predicted simultaneously by all databases. Therefore, we explored miR-29c-3p expression by qRT-PCR. As illustrated in Figure 1E and F, miR-29c-3p was downregulated both in colonic tissues and IECs from UC patients relative to normal controls. Subsequently, as illustrated by Pearson correlation scatter plots (Figure $1 \mathrm{G}$ and $\mathrm{H}$ ), miR-29c-3p and LIF protein expression in UC tissues were inversely correlated, whereas disparity was found between miR-29c-3p and LIF mRNA expression. Accordingly, we hypothesized that miR-29c-3p is involved in the post-transcriptional regulation of LIF expression.

\section{Identification of Human Colonic LIF as a Direct Target of miR-29c-3p}

Given the inverse correlation between miR-29c-3p and LIF protein expression, we next investigated whether LIF is a putative target of miR-29c-3p using TargetScan software. As shown in Figure 2A, a putative binding site of miR-29c-3p in the $3^{\prime}$-UTR of human LIF was identified. For further

Table I The Primer Sequences for qRT-PCR

\begin{tabular}{|l|l|l|}
\hline Gene & Forward & Reverse \\
\hline Human LIF & CCAACGTGACGGACTTCCC & TACACGACTATGCGGTACAGC \\
Mouse LIF & ATTGTGCCCTTACTGCTGCTG & GCCAGTTGATTCTTGATCTGGT \\
Human $\beta$-actin & CATGTACGTTGCTATCCAGGC & CTCCTTAATGTCACGCACGAT \\
Mouse $\beta$-actin & AGTGTGACGTTGACATCCGTA & GCCAGAGCAGTAATCTCCTTCT \\
\hline
\end{tabular}




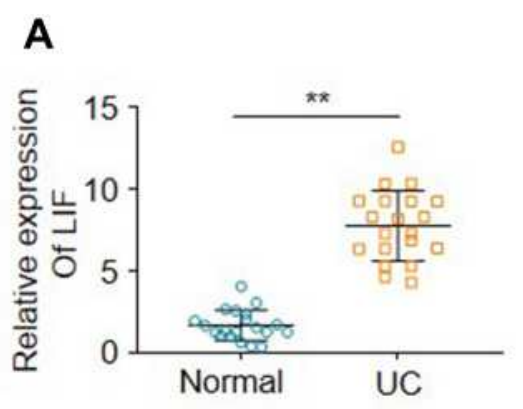

C

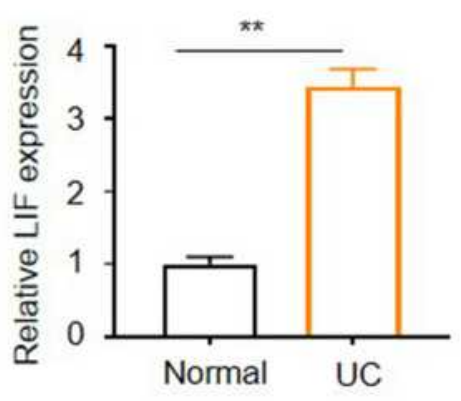

E

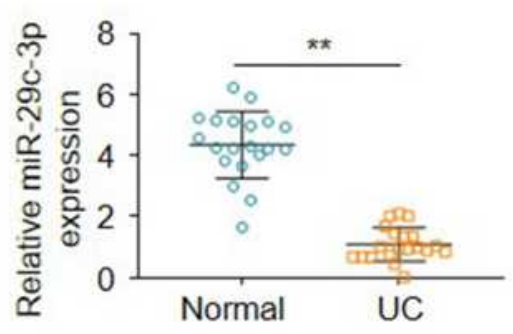

G

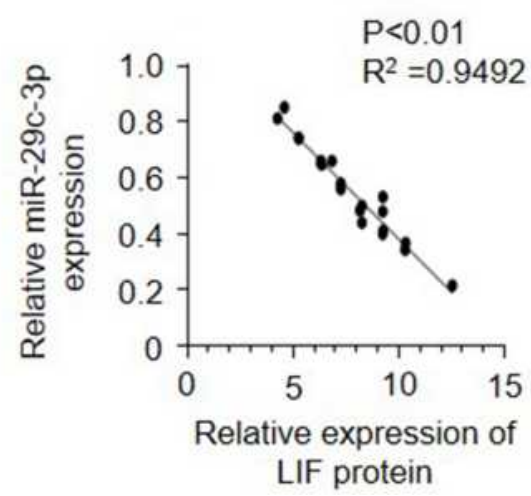

B
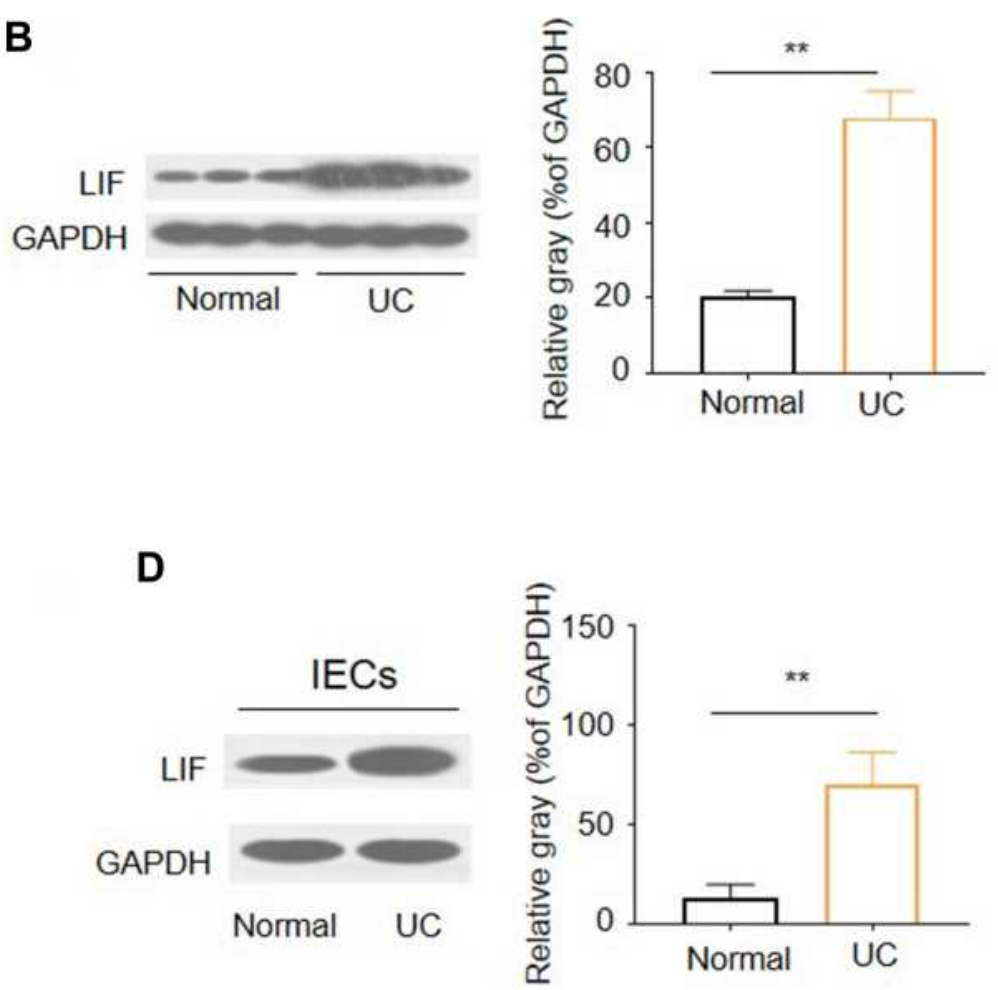

F

IECs

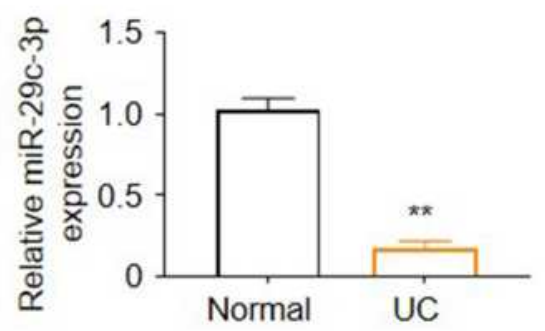

H

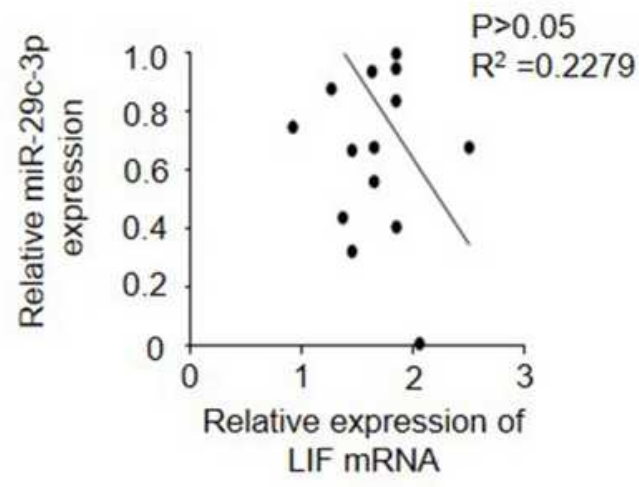

Figure I The expression of miR-29c-3p and LIF in UC tissues and IECs. (A) The mRNA levels of LIF in UC and normal tissues. (B) Representative Western blots and densitometric quantitative analysis of LIF proteins levels in UC and normal tissues. (C) The mRNA levels of LIF in primary IECs of UC and normal colon. (D) Representative Western blots and densitometric quantitative analysis of LIF protein levels in IECs of UC and normal colon. (E) The expression of miR-29c-3p in UC and normal tissues. (F) The expression of miR-29c-3p in primary IECs of UC and normal colon. (G) Pearson's correlation analysis of the expression levels of miR-29c-3p and LIF protein. (H) Pearson's correlation analysis of the expression levels of miR-29c-3p and LIF mRNA. $* * P<0.0$ I versus normal group. 
A

LIF-wt 5'----UUUAUUAACUUAUUGGUGCUU-3'

miR-29c-3p 3'-AUUGGCUAAAGUUU----ACCACGAU_-5'

LIF-mut 5'- CAAAUUCCUCUUUGAACUUACAA-3'
B

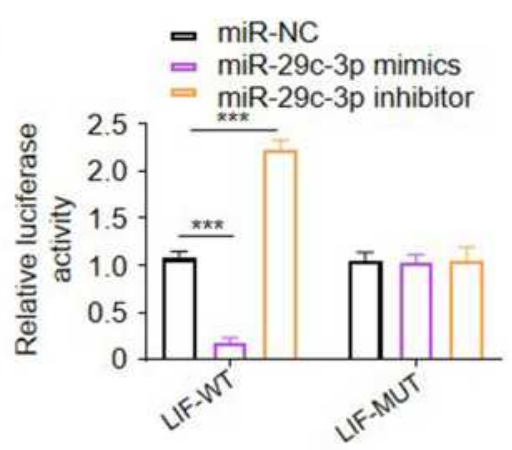

C

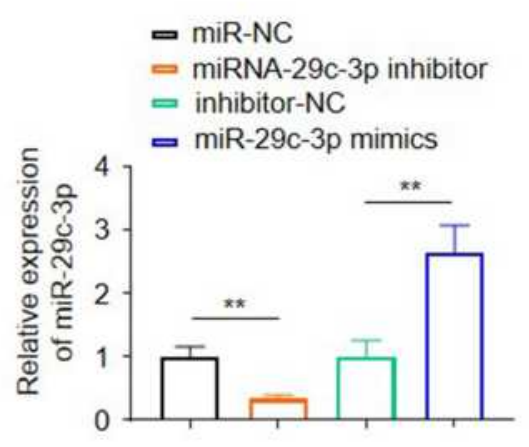

E

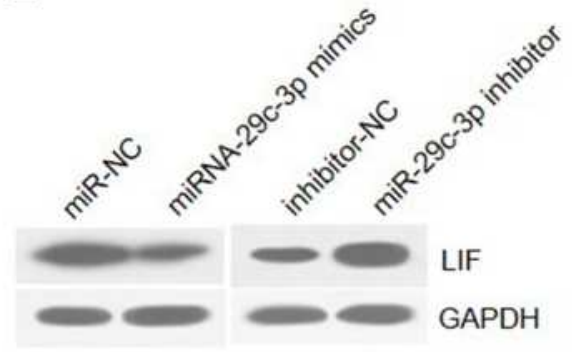

D
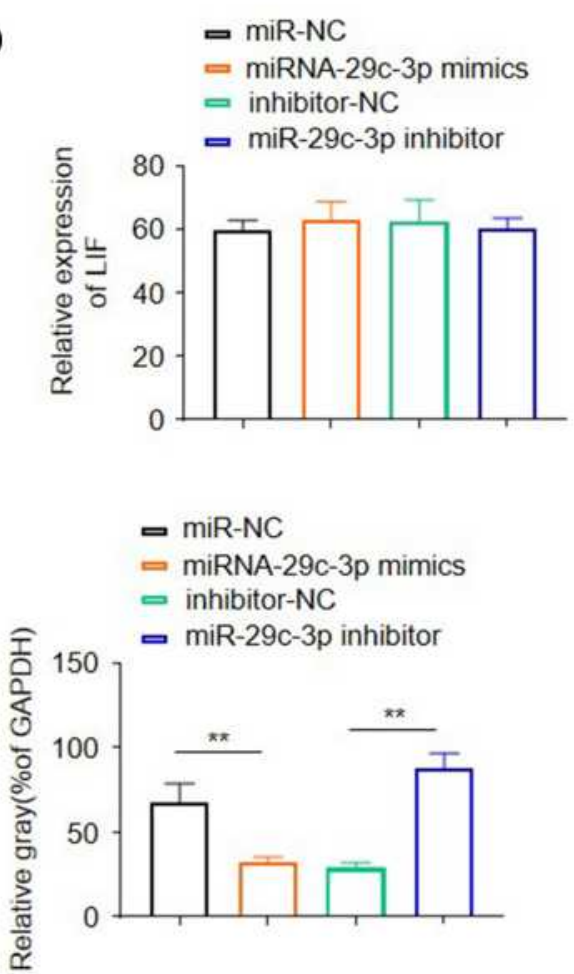

Figure 2 Identification of LIF as a direct target of miR-29c-3p. (A) Schematic description of a putative binding site of miR-29c-3p in the 3'-UTR of human LIF. (B) Luciferase reporter activity in Caco-2 cells. (C) QRT-PCR analysis of miR-29c-3p expression in Caco-2 cells pre-transfected with miR-29c-3p mimics, miR-29c-3p inhibitor or corresponding scramble controls. (D) qRT-PCR analysis of LIF mRNA levels in pre-transfected Caco-2 cells. (E) Representative Western blots and densitometric quantitative analysis of LIF protein levels in pre-transfected Caco- 2 cells. ${ }^{* *}$ and $* * *$ indicates $\mathrm{P}<0.0 \mathrm{I}$ and $0.00 \mathrm{I}$, respectively.

validation, a luciferase reporter assay was conducted to determine whether miR-29c-3p directly targets the 3 '-UTR of human LIF mRNA. The results showed that co-transfected with 3'-UTR luciferase reporter and miR-29c-3p mimic or inhibitor in Caco-2 cells resulted in a remarkable decrease or increase in luciferase reporter activity, respectively, compared to scrambled control and 3'-UTR reporter co-treated cells, while no difference was found when 3 '-UTR of LIF was mutated (Figure 2B).

To uncover the post-transcriptional regulation of miR$29 \mathrm{c}-3 \mathrm{p}$ on human LIF, we detected the mRNA and protein levels of LIF by overexpression and knockdown of miR- 29c-3p in Caco-2 cells. As shown in Figure $2 \mathrm{C}$ and E, transfection with miR-29c-3p mimic markedly enhanced miR-29c-3p expression while decreasing LIF protein level compared with control group. Conversely, in comparison with inhibitor-NC group, knockdown of miR-29c-3p inhibited miR-29c-3p expression but increased LIF protein levels. In contrast, alteration of miR-29c-3p intracellular level had no impact on LIF mRNA expression in Caco-2 cells (Figure 2D). Taken together, these results demonstrated that miR-29c-3p directly targets the 3'-UTR of human colonic LIF and acts as a regulator at the posttranscriptional level. 


\section{The Role of miR-29c-3p and LIF on Inflammatory Cytokines Production in vitro} It was previously demonstrated that LIF treatment protected against DSS-induced mice colitis, ${ }^{16}$ suggesting LIF confers an anti-inflammatory action on intestinal inflammation. Thus, we next focused on the role of miR-29c-3p/LIF axis in the regulation of inflammatory response. First, we investigated miR-29c$3 p$ and LIF expression in Caco-2 cells when stimulated with LPS (Figure 3A and C). We observed a decrease of miR-29c$3 p$ and an increase in LIF mRNA and protein expression in
LPS-treated cells, which was consistent with the results in UC patients.

Subsequently, we wondered whether miR-29c-3p can regulate inflammatory response via modulating LIF in cells challenged with LPS. As shown in Figure 3A and B, miR-29c-3p mimics transfection successfully upregulated miR-29c-3p expression in LPS-stimulated Caco-2 cells and resulted in a decline of LIF protein levels. However, no significant alteration was found on LIF mRNA expression after miR-29c-3p overexpression (Figure 3C), which was conformed to our
A
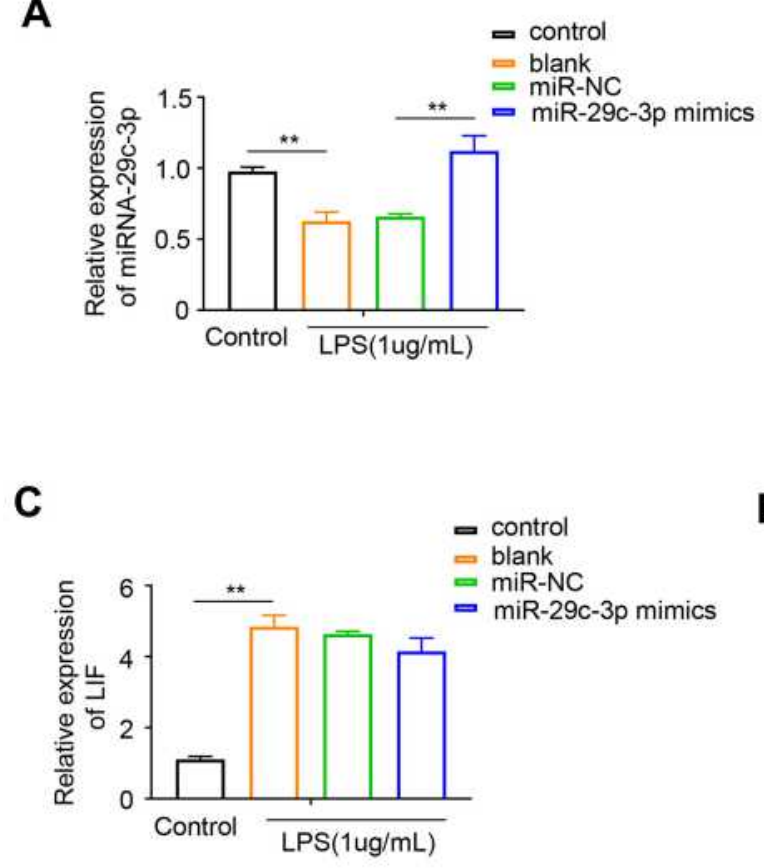

B

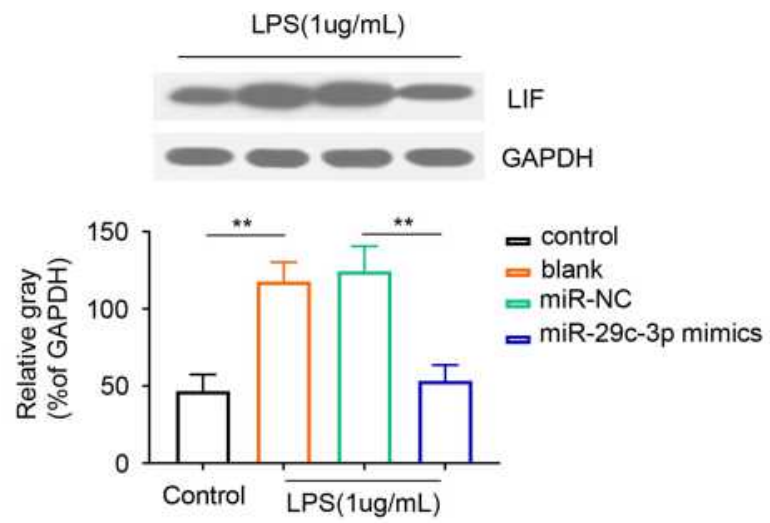

D
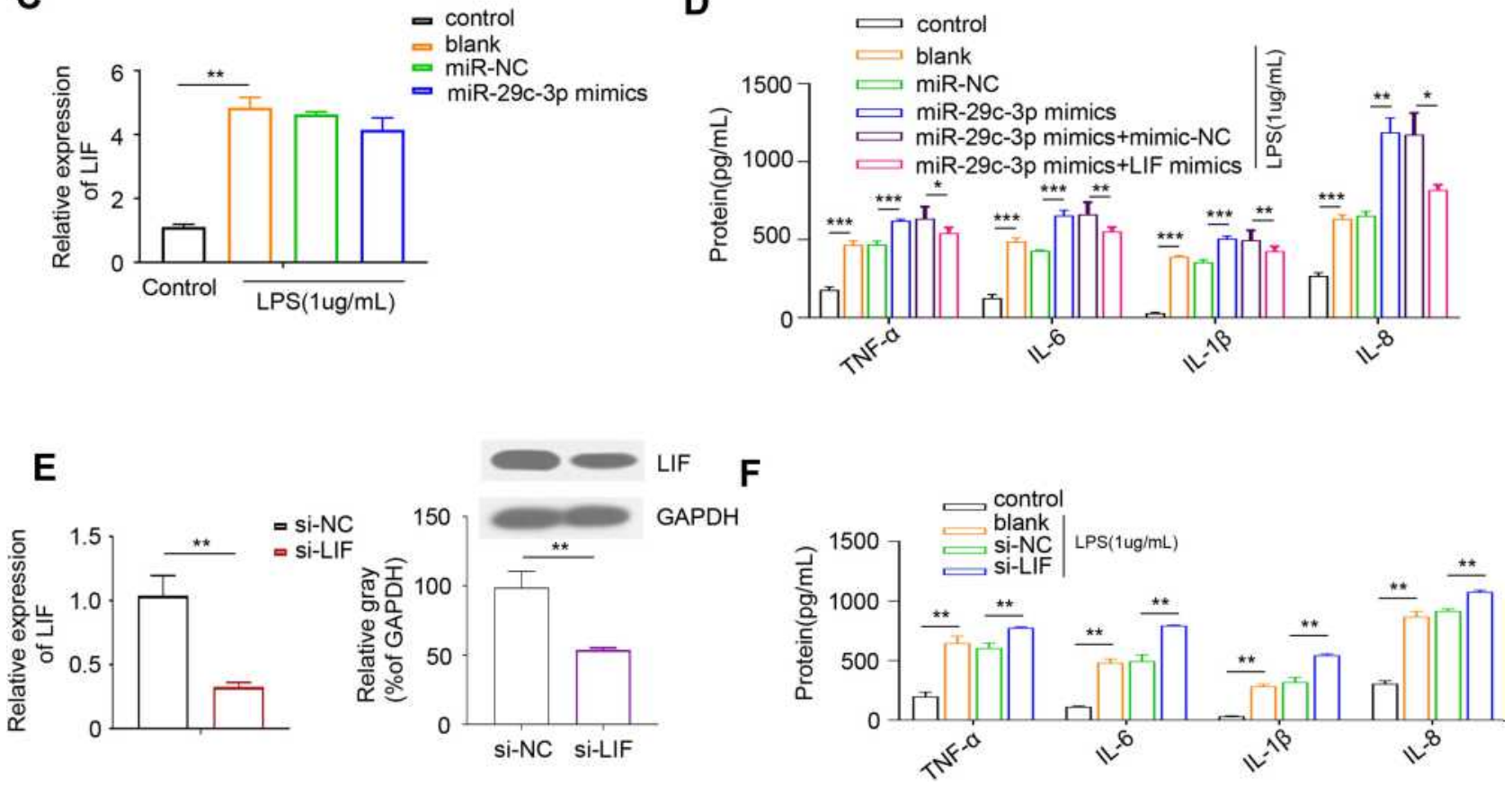

Figure 3 The role of miR-29c-3p and LIF in LPS-mediated cell inflammation. (A) The expression of miR-29c-3p in LPS-treated Caco-2 cells pre-transfected with miR-29c-3p mimics or scrambled control. (B) Representative Western blots and densitometric quantitative analysis of LIF protein levels in LPS-treated Caco-2 cells pre-transfected with miR-29c-3p mimics or scrambled control. (C) qRT-PCR analysis of LIF expression in LPS-treated Caco-2 cells pre-transfected with miR-29c-3p mimics or scrambled control. (D) The protein levels of IL-I $\beta$, IL-6, IL-8 and TNF- $\alpha$ in the culture supernatants of LPS-treated Caco-2 cells pre-transfected with miR-29c-3p mimics or together with LIF mimics. (E) Validation of si-LIF transfection efficiency in Caco-2 cells. (F) The protein levels of IL-I $\beta$, IL-6, IL- 8 and TNF- $\alpha$ in the culture supernatants of LPS-treated Caco-2 cells pre-transfected with si-LIF or scrambled control. $* \mathrm{P}<0.05$, $* * \mathrm{P}<0.0 \mathrm{I}, * * * \mathrm{P}<0.00 \mathrm{I}$. 
hypothesis that miR-29c-3p alters the translational process of LIF.

To further investigate the role of miR-29c-3p in modulating cell inflammation, we tested the levels of several proinflammatory cytokines in the culture supernatants of Caco-2 cells by ELISA. As a result, the upregulation of miR-29c-3p observably increased, while the co-transfection of miR-29c-3p and LIF mimics reversed the production of IL-1 $\beta$, IL-6, IL-8 and TNF- $\alpha$ (Figure 3D). Likewise, silencing of LIF in Caco-2 cells dramatically suppressed LIF expression and augmented the secretion of the above four cytokines (Figure 3E and F). Taken together, our findings revealed that miR-29c-3p overexpression inhibits LIF protein expression, and thereby promotes the LPS-induced production of pro-inflammatory mediators.

\section{The Role of miR-29c-3p and LIF on Cell Proliferation and Apoptosis}

MiR-29c-3p inhibited the proliferation of HCT116 cells as previously reported. ${ }^{17}$ Therefore, we next investigated the role of miR-29c-3p/LIF axis in cell proliferation and apoptosis. For cell proliferation examination, CCK-8 assay was carried out in Caco-2 cells. As illustrated in Figure 4A and $\mathrm{C}$, the overexpression of miR-29c-3p and LIF silencing
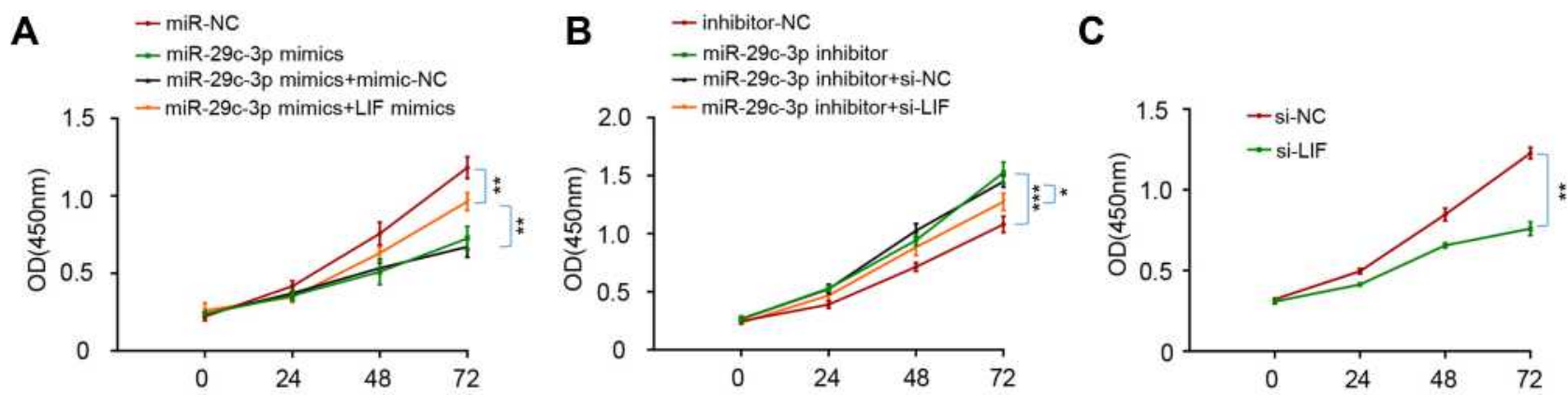

D
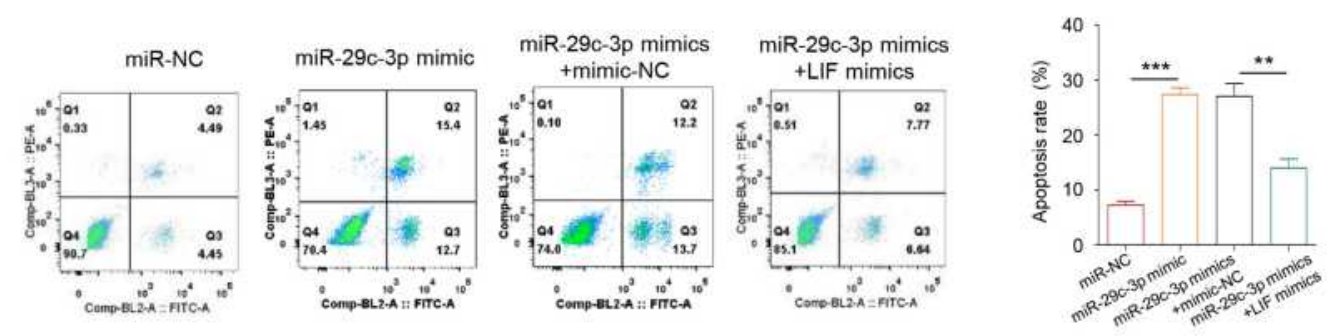

$\mathbf{E}$
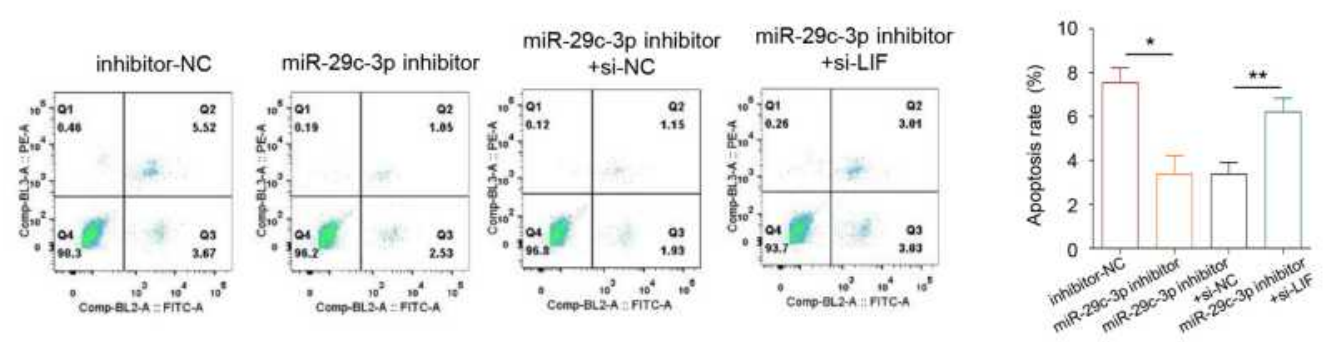

$\mathbf{F}$
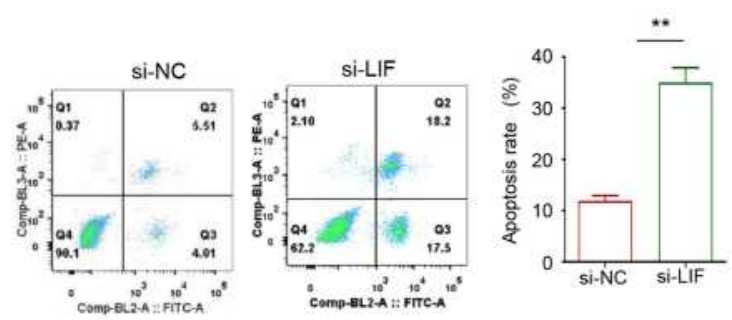

Figure 4 The role of miR-29c-3p and LIF on cell proliferation and apoptosis. (A) CCK-8 assays of Caco-2 cells after transfection with miR-29c-3p mimics and LIF mimics. (B) CCK8 assays of Caco-2 cells after transfection with miR-29c-3p inhibitor and si-LIF. (C) CCK-8 assays of Caco-2 cells after transfection with si-LIF or scrambled control. (D)Flow cytometry analysis of cell apoptosis after transfection with miR-29c-3p mimics and LIF mimics (E) Flow cytometry analysis of cell apoptosis after transfection with miR-29c-3p inhibitor and si-LIF. (F) Flow cytometry analysis of cell apoptosis after transfection with si-LIF or scrambled control. $* \mathrm{P}<0.05, * * \mathrm{P}<0.0 \mathrm{I}, * * * \mathrm{P}<0.00 \mathrm{I}$. 
suppressed cell proliferation ability, whereas it was rescued by LIF mimics treatment. Unsurprisingly, the knockdown of miR-29c-3p exerted the opposite effects, which was partially inhibited by co-transfection of si-LIF (Figure 4B). In addition, an increase in apoptotic cells was identified in miR-29c-3p overexpression and LIF knockdown cells, while cell apoptosis was depressed by miR-29c-3p silencing (Figure 4D and F). Similarly, the effects of miR-29c-3p could be rescued by LIF overexpression or silencing, suggesting the modulatory functions of miR-29c-3p/LIF axis on cell apoptosis.

\section{MiR-29c-3p Mimics Treatment Aggravates DSS-Induced Colitis via Suppressing LIF}

First, we determined that miR-29c-3p and LIF were differentially expressed in DSS-induced murine colitis. As demonstrated in Figure 5A and B, miR-29c-3p level was markedly decreased in both inflamed tissues and primary IECs after DSS administration, while LIF protein level was significantly increased, which accorded with the results in human samples. Subsequently, we treated colitis mice with miR-29c-3p mimic intracolonically to identify the pro-inflammatory effect of miR-29c-3p on gut inflammation. As a result, miR-29c-3p was upregulated but LIF protein was decreased after miR-29c-3p mimic treatment. In contrast, although LIF mRNA level was induced by DSS, miR-29c-3p mimic just slightly lowered LIF mRNA expression but without statistically significance (Figure 5C). Subsequently, locally overexpression of miR-29c-3p resulted in an exacerbation of colitis as determined by weight loss, colon length, DAI scores, macroscopy and histopathological evaluation. Colitis mice administered with miR-29c-3p mimic suffered dramatic weight loss compared to other groups (Figure 6A). The average colon length of mice in DSS+PEI/miRNA$29 c-3 p$ mimic group was much shorter (Figure 6B and C), and apparently the mice had higher DAI scores after miRNA-29c-3p mimic treatment (Figure 6D). As shown by histopathological examination, we observed severe tissue damages in DSS treated mice as reflected by crypt destruction and inflammatory cells infiltration in epithelium and lamina propria, whereas miRNA-29c-3p mimic administration even aggravated the colonic structural damages (Figure 6E and $\mathrm{F}$ ). In addition, the levels of pro-inflammatory factors (IL- $1 \beta$, IL-6, IL-8 and TNF- $\alpha$ ) in inflamed colon tissues were ulteriorly increased after overexpression of miRNA-29c-3p (Figure 6G). In general, mice treated with miRNA-29c-3p mimic experienced a suppression of LIF and developed more severe colitis.

\section{Discussion}

IL-6 related cytokines have been considered to play pivotal roles in the orchestration of immune progression in a variety of inflammatory-immune diseases. ${ }^{20}$ As a member of IL-6 cytokine family, LIF exerted a protective function in experimental colitis by inhibiting Th17 cells differentiation and promoting epithelium reconstruction as described previously. ${ }^{16}$ However, there are few studies regarding on the specific effect of LIF in IBD, and the upstream regulation of LIF in colonic inflammation is largely unknown. Given that dysregulation of miRNAs has been linked to the pathogenesis of IBD, ${ }^{21-24}$ herein we sought to explore the putative upstream miRNA of LIF and the underlying effect of miRNA/LIF axis in vivo and in vitro.

According to our findings, LIF expression was prominently increased in UC patients, which further confirmed previous research results. ${ }^{15}$ Then, we predicted miR-29c$3 p$ as the upstream miRNA of human LIF through five bioinformatics software, and the conserved binding site in 3'-UTR of human LIF was substantiated by luciferase reporter assay. As a member of miR-29 family, miR-29c$3 p$ has been reported to be downregulated and attributed predominantly anti-tumor properties in multiple human cancers, including colorectal cancer. ${ }^{17,25}$ Nevertheless, the expression of miR-29c-3p in IBD is still uncovered, thereby we examined it in UC patients and a murine colitis model mimicking human UC. As expected, a decrease of miR-29c-3p was found in UC colon tissues, which was inversely correlated with LIF protein but not LIF mRNA levels. Therefore, miR-29c-3p possibly modulates LIF expression at the post-transcriptional level, according to the canonical regulation mechanisms of miRNA. Subsequently, this hypothesis was verified by the overexpression and knockdown of miR-29c-3p in cell lines.

IECs are indispensable components in maintaining gut homeostasis and regulating intestinal immunity. IECs not only act as a physical barrier to prevent invasive foreign pathogens but also function as an immunological barrier to mediate the crosstalk between immune cells and commensals. $^{26,27}$ As reported previously, LIF was markedly elevated in the inflamed IECs from mice with colitis and miR-29c-3p expression was lower in the human colon cancer cell-line HCT116. ${ }^{16,17}$ In our study, we also found an increase of LIF and a decrease of miR-29c-3p in the 
A

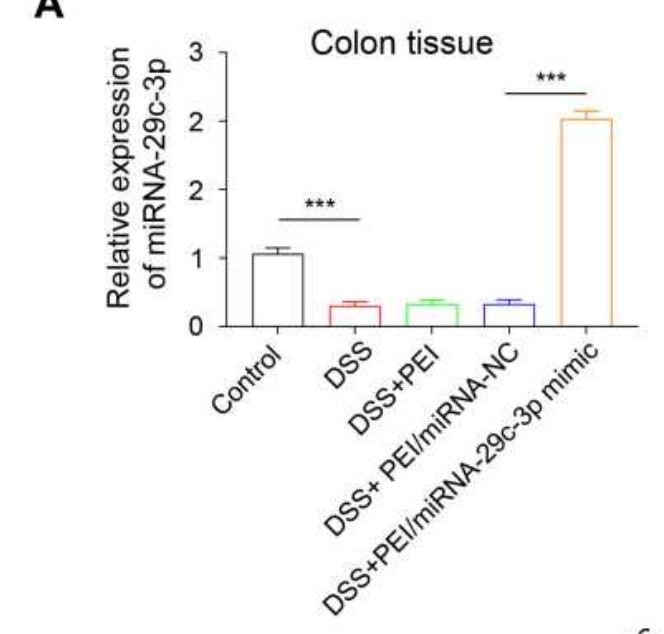

B

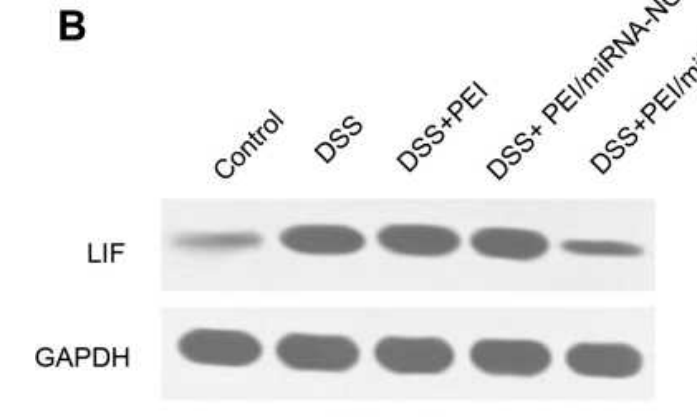

Colon tissue

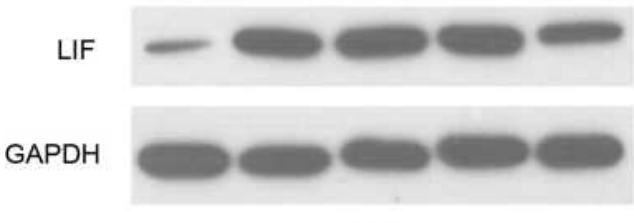

IECs

C
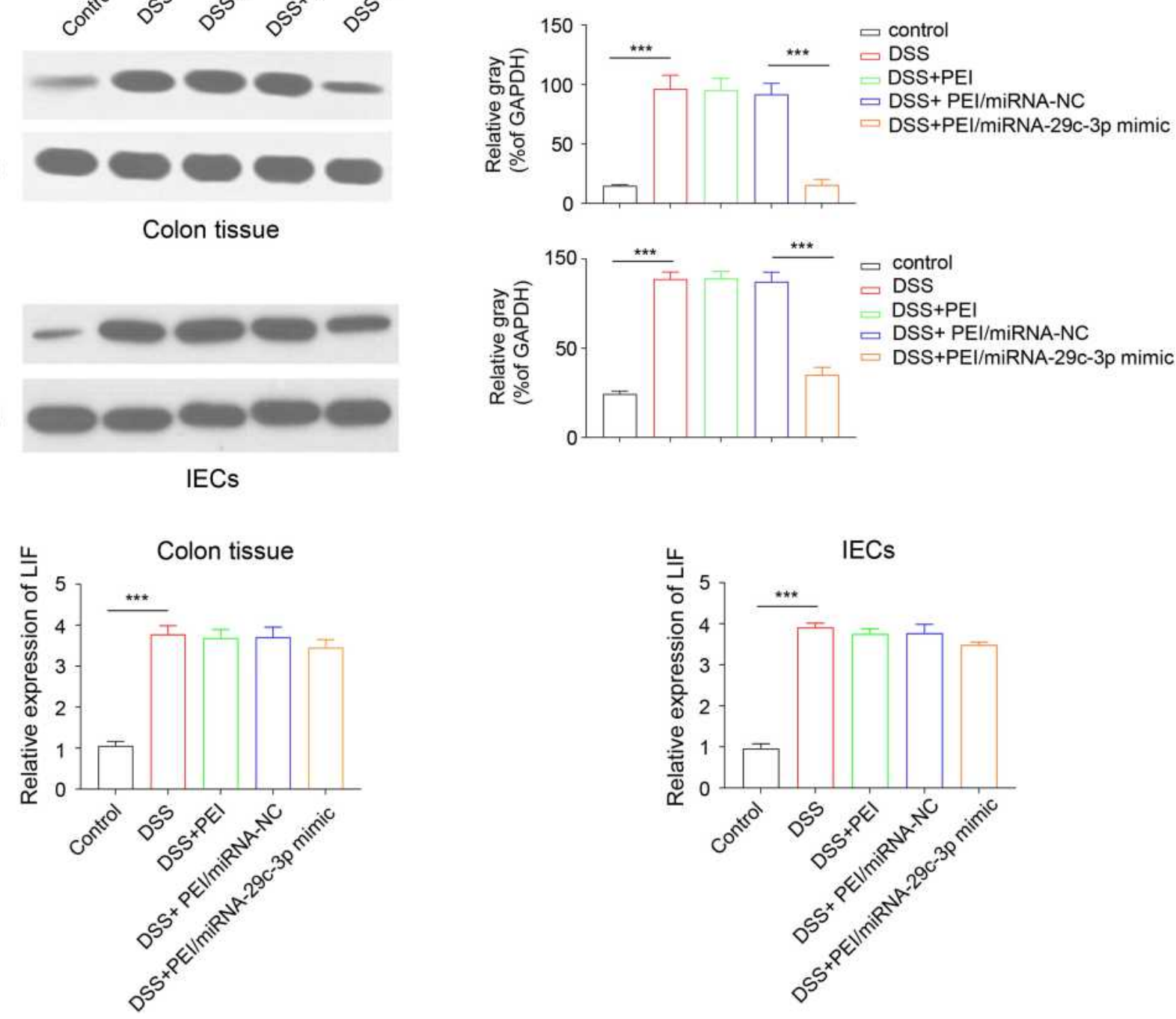

Figure 5 The expression of miR-29c-3p and LIF in DSS-induced mice colitis. Mice were given 2.5\% DSS in the drinking water for consecutive 7 days to induce experimental colitis. (A) The expression of miR-29c-3p in colon tissues and primary IECs of mice. (B) Representative Western blots and densitometric quantitative analysis of LIF protein levels in colon tissues and primary IECs. (C) qRT-PCR analysis of LIF expression in colon tissues and primary IECs. ** and *** indicates P<0.0I and $0.00 \mathrm{I}$, respectively.

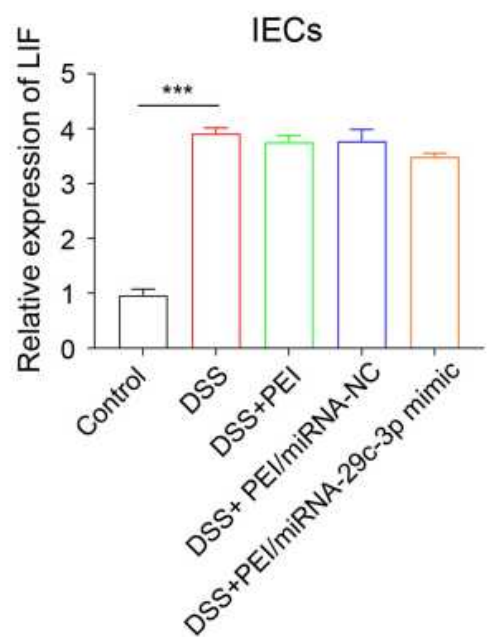




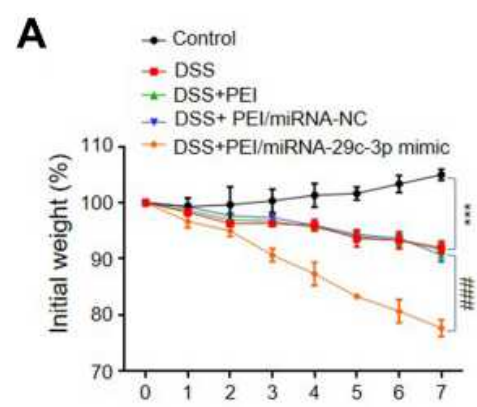

C

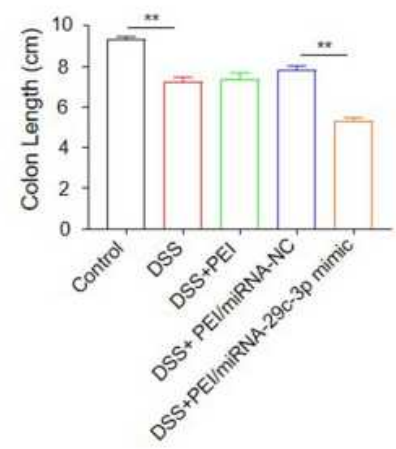

E
B

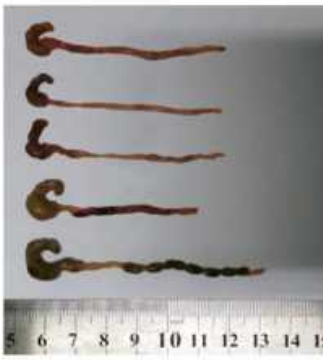

D
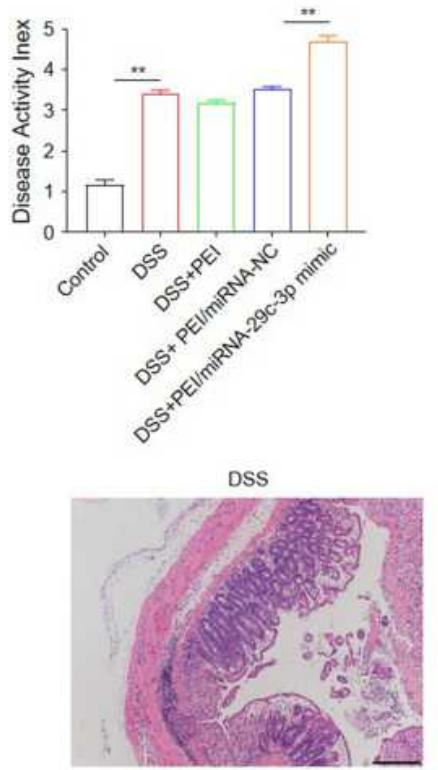

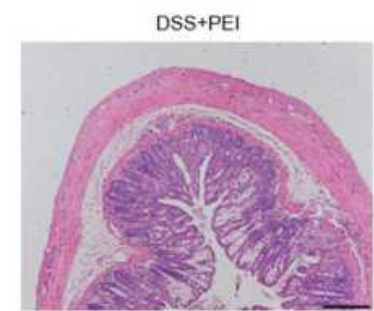

$\mathbf{F}$

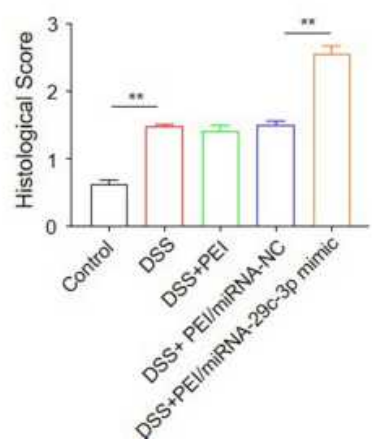

DSS

DSS+PEI

DSS+PEI/miRNA-NC

DSS+PEI/miRNA-29c-3p mimic

Control

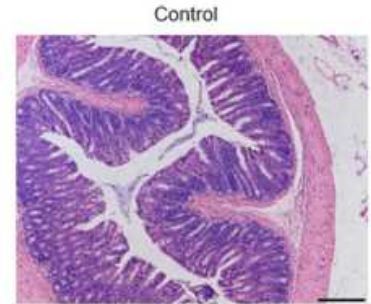

E

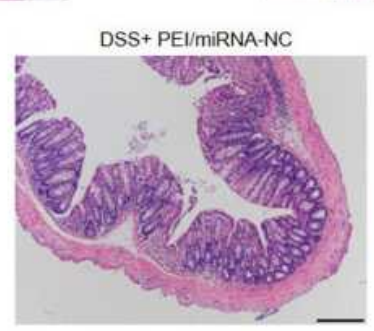

G
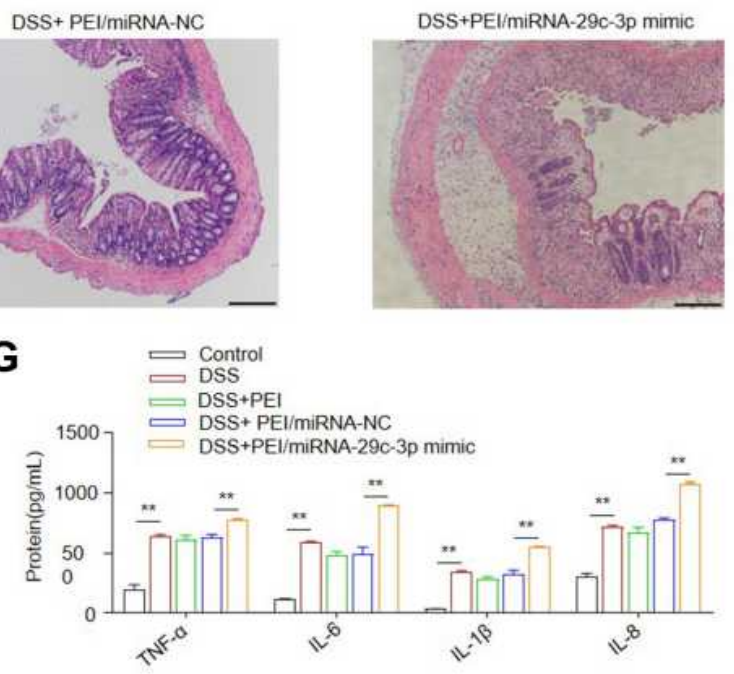

Figure 6 MiR-29c-3p mimics treatment aggravates DSS-induced mice colitis. DSS-treated mice were intracolonically introduced with miR-29c-3p mimics. Mice were euthanatized 7 days after DSS treatment and the severity of colitis was evaluated. (A) Changes of body weight of mice of different groups are shown as the percentage of initial weight. $* * *$ Indicates $\mathrm{P}<0.001$, DSS group versus control group. ${ }^{\prime \prime \prime I n d i c a t e s ~} \mathrm{P}<0.001$, DSS+PEI/miR-29c-3p mimics group versus DSS+PEI/miR-NC group. (B) Colon gross morphology of different groups. (C) Colon length in the indicated groups. (D) The disease activity index (DAI). (E) Macroscopic evaluation of colon inflammation. X200 Magnification. (F) The histological scores. (G) The protein levels of IL-I $\beta$, IL-6, IL-8 and TNF- $\alpha$ in the colon tissues of mice in different groups. $* *$ and $* * *$ indicates $\mathrm{P}<0.0 \mathrm{I}$ and $0.00 \mathrm{I}$, respectively. 
IECs extracted from human and mice inflamed colon tissues.

To further investigate the underlying mechanisms of miR-29c-3p/LIF axis in UC, we adopted LPS-stimulated Caco-2 cells as a cell inflammatory model. LPS challenge leads to an elevation of pro-inflammatory mediators by interacting with toll-like receptors (TLRs). ${ }^{28}$ Nguyen et al stated that an autocrine feedback loop involving LIF, LIFR and STAT4 promoted sustained inflammatory cytokines production by fibroblasts. ${ }^{29}$ However, LIF may exert either pro- or anti-inflammatory function in different cell types and diseases, depending on a number of variables. $^{30}$ As expected, similar results for LIF and miR-29c-3p expression profile were identified in inflamed Caco- 2 cells induced by LPS stimulation. Besides, miR29c-3p overexpression and LIF silencing both ulteriorly enhanced the production of IL- $1 \beta$, IL-6, IL-8 and TNF- $\alpha$ in Caco-2 cells after LPS treatment. These data suggest that miR-29c-3p exerts a pro-inflammatory effect on LPSinduced inflammation via inhibiting LIF expression, thereby targeting miR-29c-3p that provides a promising anti-inflammation therapy.

In a previous study, miR-29c-3p showed an effect on HCT116 cells migration and proliferation. ${ }^{17}$ LIF was also reported to promote IECs proliferation. ${ }^{16}$ In another study, LIF deficiency impaired the renewal and shortened the lifespan of intestinal epithelium, which could be rescued by LIF administration. $^{31}$ In the current study, we investigated the effect of miR-29c-3p/LIF axis on cell proliferation and apoptosis. We found that overexpression of miR-29c-3p inhibited Caco- 2 cells proliferation and drove cell apoptosis via suppressing LIF, while knockdown of miR-29c-3p showed totally opposite effects.

The current data of in vitro studies indicated that miR-29c$3 p$ played a pro-inflammatory role in gut inflammation, whereas LIF acted as a protective factor. To further confirm the results, we established a DSS-induced murine colitis model. MiR-29c-3p expression was decreased and LIF was increased in DSS treated mice, which was consistent with the results in UC patients. After intracolonical injection with miR$29 \mathrm{c}-3 \mathrm{p}$ mimic, LIF protein level was downregulated as expected, and mice developed with exacerbated manifestations of colitis. In addition, the levels of pro-inflammatory cytokines (IL-1 $\beta$, IL-6, IL-8 and TNF- $\alpha$ ) was significantly elevated in inflamed colon, while miR-29c-3p mimic treatment further enhanced cytokines production. Thus, experiments on the murine colitis model validated the pro-inflammatory effect of miR-29c-3p.
In summary, we first demonstrate that miR-29c-3p/LIF axis plays an important role in the regulation of intestinal inflammation. Overexpression of miR-29c-3p promotes inflammation via suppressing LIF both in vitro and in vivo. The differential expression profile of miR-29c-3p and LIF in inflamed colon lesions may provide a promising biomarker for UC. MiR-29c-3p/LIF pathway serves as a potential therapeutic target for UC treatment.

\section{Data Sharing Statement}

The datasets generated during and analyzed during the current study are available from the corresponding authors on reasonable request.

\section{Author Contributions}

All authors made substantial contributions to conception and design, acquisition of data, analysis and interpretation of data; took part in drafting the article or revising it critically for important intellectual content; agreed to submit to the current journal; gave final approval of the version to be published; and agree to be accountable for all aspects of the work.

\section{Funding}

This research did not receive any specific grant from funding agencies in the public, commercial, or not-forprofit sectors.

\section{Disclosure}

The authors declare that there are no conflicts of interest.

\section{References}

1. Itzkowitz S. Colon carcinogenesis in inflammatory bowel disease: applying molecular genetics to clinical practice. $J$ Clin Gastroenterol. 2003;36(5Suppl):S70-S74. doi:10.1097/00004836200305001-00012

2. Xie C, Yan W, Quan R, et al. Interleukin-38 is elevated in inflammatory bowel diseases and suppresses intestinal inflammation. Cytokine. 2020;127:154963. doi:10.1016/j.cyto.2019.154963

3. Bamias G, Corridoni D, Pizarro TT, Cominelli F. New insights into the dichotomous role of innate cytokines in gut homeostasis and inflammation. Cytokine. 2012;59(3):451-459. doi:10.1016/j.cyto.2012.06.014

4. Dubois-Camacho K, Ottum PA, Franco-Muñoz D, et al. Glucocorticosteroid therapy in inflammatory bowel diseases: from clinical practice to molecular biology. World $J$ Gastroenterol. 2017;23(36):6628. doi:10.3748/wjg.v23.i36.6628

5. Doench JG, Sharp PA. Specificity of microRNA target selection in translational repression. Genes Dev. 2004;18(5):504-511. doi:10.1101/ gad.1184404

6. Gebert LF, MacRae IJ. Regulation of microRNA function in animals. Nat Rev Mol Cell Biol. 2019;20(1):21-37.

7. Calin GA, Croce CM. MicroRNA signatures in human cancers. Nat Rev Cancer. 2006;6(11):857-866. doi:10.1038/nrc1997 
8. Coskun M, Bjerrum JT, Seidelin JB, Nielsen OH. MicroRNAs in inflammatory bowel disease-pathogenesis, diagnostics and therapeutics. World J Gastroenterol. 2012;18(34):4629-4634. doi:10.3748/ wjg.v18.i34.4629

9. Esteller M. Non-coding RNAs in human disease. Nat Rev Genet. 2011;12(12):861-874. doi:10.1038/nrg3074

10. Kloosterman WP, Plasterk RH. The diverse functions of microRNAs in animal development and disease. Dev Cell. 2006;11(4):441-450. doi:10.1016/j.devcel.2006.09.009

11. O'Connell RM, Rao DS, Baltimore D. microRNA regulation of inflammatory responses. Annu Rev Immunol. 2012;30:295-312. doi:10.1146/annurev-immunol-020711-075013

12. Tian $\mathrm{Y}, \mathrm{Xu} \mathrm{J}, \mathrm{Li} \mathrm{Y}$, et al. MicroRNA-31 reduces inflammatory signaling and promotes regeneration in colon epithelium, and delivery of mimics in microspheres reduces colitis in mice. Gastroenterology. 2019;156(8):2281-2296.e2286. doi:10.1053/j. gastro.2019.02.023

13. He C, Shi Y, Wu R, et al. miR-301a promotes intestinal mucosal inflammation through induction of IL-17A and TNF- $\alpha$ in IBD. Gut. 2016;65(12):1938-1950. doi:10.1136/gutjnl-2015-309389

14. Nicola NA, Babon JJ. Leukemia inhibitory factor (LIF). Cytokine Growth Factor Rev. 2015;26(5):533-544. doi:10.1016/j. cytogfr.2015.07.001

15. Guimbaud R, Abitbol V, Bertrand V, et al. Leukemia inhibitory factor involvement in human ulcerative colitis and its potential role in malignant course. Eur Cytokine Netw. 1998;9(4):607-612.

16. Zhang YS, Xin DE, Wang Z, et al. STAT 4 activation by leukemia inhibitory factor confers a therapeutic effect on intestinal inflammation. EMBO J. 2019;38(6):1-20. doi:10.15252/ embj.201899595

17. Zhang S, Jin J, Tian X, Wu L. hsa-miR-29c-3p regulates biological function of colorectal cancer by targeting SPARC. Oncotarget 2017;8(61):104508-104524. doi:10.18632/oncotarget.22356

18. Grossmann J, Maxson JM, Whitacre CM, et al. New isolation technique to study apoptosis in human intestinal epithelial cells. Am J Pathol. 1998;153(1):53-62. doi:10.1016/S0002-9440(10)65545-9

19. Cooper HS, Murthy S, Shah R, Sedergran D. Clinicopathologic study of dextran sulfate sodium experimental murine colitis. Lab Invest. 1993;69(2):238.

20. Jones SA, Jenkins BJ. Recent insights into targeting the IL-6 cytokine family in inflammatory diseases and cancer. Nat Rev Immunol. 2018;18(12):773-789.
21. Bian Z, Li L, Cui J, et al. Role of miR-150-targeting c-Myb in colonic epithelial disruption during dextran sulphate sodium-induced murine experimental colitis and human ulcerative colitis. J Pathol. 2011;225(4):544-553. doi:10.1002/path.2907

22. Takagi T, Naito Y, Mizushima K, et al. Increased expression of microRNA in the inflamed colonic mucosa of patients with active ulcerative colitis. J Gastroenterol Hepatol. 2010;25:S129-S133. doi:10.1111/j.1440-1746.2009.06216.x

23. Wu F, Zhang S, Dassopoulos T, et al. Identification of microRNAs associated with ileal and colonic Crohn's disease. Inflamm Bowel Dis. 2010;16(10):1729-1738. doi:10.1002/ibd.21267

24. Wu F, Zikusoka M, Trindade A, et al. MicroRNAs are differentially expressed in ulcerative colitis and alter expression of macrophage inflammatory peptide-2 $\alpha$. J Gastroenterol. 2008;135(5):1624-1635. doi:10.1053/j.gastro.2008.07.068

25. Schmitt MJ, Margue C, Behrmann I, Kreis S. MiRNA-29: a microRNA family with tumor-suppressing and immune-modulating properties. Curr Mol Med. 2013;13 (4):572-585. doi:10.2174/1566524011313040009

26. Goto Y, Ivanov II. Intestinal epithelial cells as mediators of the commensal-host immune crosstalk. Immunol Cell Biol. 2013;91 (3):204-214. doi:10.1038/icb.2012.80

27. Kayama H, Takeda K. Regulation of intestinal homeostasis by innate and adaptive immunity. Int Immunol. 2012;24(11):673-680. doi:10.1093/intimm/dxs094

28. Lu YC, Yeh WC, Ohashi PS. LPS/TLR4 signal transduction pathway. Cytokine. 2008;42(2):145-151. doi:10.1016/j.cyto.2008.01.006

29. Nguyen HN, Noss EH, Mizoguchi F, et al. Autocrine loop involving IL-6 family member LIF, LIF receptor, and STAT4 drives sustained fibroblast production of inflammatory mediators. Immunity. 2017;46 (2):220-232. doi:10.1016/j.immuni.2017.01.004

30. Knight D. Leukaemia inhibitory factor (LIF): a cytokine of emerging importance in chronic airway inflammation. Pulm Pharmacol Ther. 2001;14(3):169-176. doi:10.1006/pupt.2001.0282

31. Wang H, Wang J, Zhao Y, et al. LIF is essential for ISC function and protects against radiation-induced gastrointestinal syndrome. Cell Death Dis. 2020;11(7):588. doi:10.1038/s41419-020-02790-6
Journal of Inflammation Research

\section{Publish your work in this journal}

The Journal of Inflammation Research is an international, peerreviewed open-access journal that welcomes laboratory and clinica findings on the molecular basis, cell biology and pharmacology of inflammation including original research, reviews, symposium reports, hypothesis formation and commentaries on: acute/chronic inflammation; mediators of inflammation; cellular processes; molecula mechanisms; pharmacology and novel anti-inflammatory drugs; clinical conditions involving inflammation. The manuscript management system is completely online and includes a very quick and fair peerreview system. Visit http://www.dovepress.com/testimonials.php to read real quotes from published authors. 